\title{
Ansiedad y depresión en pacientes obesos mórbidos: efectos a corto plazo de un programa orientado a la disminución de la sintomatología
}

\section{Anxiety and depression in morbid obesity patients: Short-term effect of a program oriented to the diminution of the symptomatology}

\author{
Alfonso Cofré Pamela Angulo-Díaz \\ Universidad Santo Tomás \\ Enrique Riquelme-Mella \\ Universidad Católica de Temuco
}

(Rec: marzo 2014 - Acep: junio 2014)

\begin{abstract}
Resumen
La obesidad mórbida es, por sus repercusiones en la calidad de vida, salud física y psicológica, uno de los principales temas de interés e investigación en el campo de la salud. La mayoría de los programas de intervención apuntan directamente a la disminución de masa corporal excluyendo o ignorando dimensiones centrales en la enfermedad, tales como la depresión o ansiedad. En este artículo pretendemos dar a conocer los efectos en las dimensiones psicológicas de un programa interdisciplinario de 10 meses de duración. Participaron 18 personas, cuyas edades oscilaron entre 20 y 60 años, $(M=39.5$; DT =9.2), 17 mujeres y 1 hombre. Todos fueron parte del programa de apoyo interdisciplinario del equipo de trabajo OBEMOB de la Universidad Santo Tomás, sede Temuco. Los resultados muestran una disminución, estadísticamente significativa, en la sintomatología ansiosa y depresiva de todos los participantes. El artículo finaliza reflexionando sobre los alcances de la intervención, las dimensiones teóricas que pueden explicar los cambios y los requerimientos de futuros programas en el área. Palabras clave: obesidad, motivación al tratamiento, psicoterapia, ansiedad, depresión.
\end{abstract}

\begin{abstract}
Morbid obesity is, by its impact on life quality and physical-psychological health, one of the main topics of interest and research in the field of health. Most intervention programs aimed directly at decreasing body mass excluding or ignoring central dimensions in the disease, such as depression or anxiety. The aim of this article was to raise awareness of the effects on the psychological dimensions of an interdisciplinary program of 10 months duration. Participated 18 persons whose ages ranged between 20 and 60 years $(M=39.5, S D=9.2), 17$ women and 1 man. All were part of the program of support interdisciplinary (multidisciplinary) team working OBEMOB from the Santo Tomas University, located in Temuco, Chile. The results show a decrease, statistically significant, in anxious and depressive symptoms of all participants. The article concludes by reflecting on the scope of the intervention, the theoretical dimensions that may explain the changes, limitations and requirements of future programs in this area.
\end{abstract}

Keywords: obesity, motivation to treatment, psychotherapy, anxiety, depression. 


\section{Factores Psicológicos asociados a la obesidad mórbida}

En este artículo se presentan -desde el punto de vista de la psicología- los efectos sobre la sintomatología ansiosa y depresiva en pacientes de la Agrupación de Obesidad Mórbida (OBEMOB) que participaron de un programa interdisciplinar. Desde el modelo denominado transteórico que ha sido complementado desde la psicoterapia constructivista, en particular el enfoque posracionalista cognitivo procesal sistémico de Vittorio Guidano (1983; 1999), desde el cual se enfatizan y propenden a los procesos de regulación emocional y autoobservación.

La obesidad ha sido considerada como la epidemia del siglo XXI, definida como un índice de masa corporal mayor a $30 \mathrm{~kg} / \mathrm{m}^{2}$. Es considerada una patología de alta prevalencia mundial y nacional que avanza de manera estable y paulatina en países desarrollados y en aquellos en vías de desarrollo (Peña \& Bacallao, 2001; Vernay et al., 2009), observándose un aumento en los índices de obesidad en la infancia y adolescencia, índices que se incrementan con la edad (Frelut, 2009), teniendo efectos significativos en la morbilidad y mortalidad tanto en jóvenes como en adultos (Kannel, D'Agistino \& Cobb, 1996; Rubenstein, 2005). En relación a lo anterior es que Halsam y James (2005), señalan que la obesidad mórbida reduce la expectativa de vida en, al menos, 7 años para las personas que ya son obesas a los 40 años de edad.

Esta patología se relaciona con el desarrollo y aumento de distintas enfermedades como por ejemplo, cardiovasculares (Sandoya et al., 2007), síndrome metabólico, diabetes, hipertensión arterial (Martínez, Rodríguez \& Martínez, 2003), insuficiencia respiratoria, hipercolesterolemia, artritis, problemas de vesícula, apnea de sueño y otros problemas psiquiátricos, psicológicos y sociales (O’Brien \& Dixon, 2002) constituyendo un problema que afecta el desarrollo individual y social.

En la descripción y explicación del problema se integran factores sociales, psicológicos, genéticos, entre otros (Moreno, 2005; Pulido-Castelblanco, Novoa-Gómez \& Muñoz-Martínez, 2013). Desde lo contextual, para Reyes, Díaz, Lera y Burrows (2011), la intensificación del consumo de alimentos con altos componentes calóricos y la reducción de la actividad física promueven un ambiente obesigénico (Komlos, Breitfelder \& Sunder, 2009; Nicklas, Baranowski, Cullen \& Berenson, 2001). Al mismo tiempo, se ha observado que el tipo y gravedad de la obesidad se encuentra directamente relacionado con las horas que el individuo permanece sentado y sin actividad física (Martínez-González, Martínez, Hu, Gibney \& Kearney, 1999).

Entre los factores psicológicos que aparecen como variables importantes en el proceso de la constitución de la obesidad y obesidad mórbida, se describen los estados ansiosos y depresivos que propician el comer en exceso (Black, Goldstein \& Mason, 2003; Ríoset al., 2008), así como una baja autoestima y pesimismo asociado a lo relevante de la dimensión corporal en la construcción de la identidad individual y social (Álvarez, 1998; Hollis, 2005), lo que se vincula además con un autoconcepto negativo, así como una pobre y menor interacción social (Elfhag, Carlsson \& Rossner, 2003; Puhl \& Heuer, 2009). Igualmente, se ha observado que la obesidad genera un efecto negativo en las personas, con una enorme carga psicológica dado por las consecuencias psicosociales que esta enfermedad conlleva (Wardle, Williamson, Johnson \& Edwards, 2006).

Finalmente, se ha evidenciado que las personas con obesidad tienen dificultades para contactarse adecuadamente con sus emociones, (De Chouly De Lenclave, Florequin \& Bailly, 2001; Ríos et al., 2008). Lo anterior implicaría limitaciones para el reconocimiento y posterior modulación afectiva, especialmente de estados emocionales negativos, favoreciendo conductas de sobrealimentación, como un modo de autorregulación emocional (Silva, 2008), existiendo un círculo vicioso entre obesidad, ansiedad y depresión que perpetua la obesidad.

El tratamiento de los síntomas afectivos (depresión, ansiedad) en pacientes obesos es de fundamental importancia, de lo contrario el paciente no será capaz de comprometerse adecuadamente con el tratamiento, y en general tendrán mayor dificultad para bajar de peso. Según Bresh (2006), muchas personas refieren que comen cuando están deprimidas y ansiosas, de manera que la ingesta de alimentos reduce ansiedades y depresiones. De igual forma Cabello y Zúñiga (2007) encontraron que la génesis de la obesidad se relaciona con los eventos de vida estresantes, por lo que se emplea la comida como refugio para compensar frustraciones, depresión, necesidades y temores que pueden además estar asociadas al prejuicio y la discriminación (Braguinsky, Markman \& Katz, 1995).

En este marco, el objetivo principal de cualquier tratamiento para la obesidad mórbida debe ser el propiciar una pérdida de peso sostenible en el tiempo, reduciendo los factores que ponen la vida del individuo 
en riesgo y mejorando el funcionamiento del individuo hacia una buena salud (Feixas \& Saldivar-Maldonado, 2010). Junto a la pérdida de peso estable, se espera que las personas que forman parte de programas multidisciplinarios para el tratamiento de la obesidad, puedan modificar factores psicológicos internos asociados al origen y mantenimiento de la enfermedad, tales como la ansiedad y la sintomatología depresiva (Frelut, 2009).

De esta forma, junto con una pérdida de peso, el objetivo del tratamiento para la obesidad mórbida debe ser una mejora en la salud que reduzca los factores que ponen la vida del individuo en riesgo y mejore su funcionamiento. Por lo tanto, se considera que el estudio y tratamiento multidisciplinar de la obesidad constituye el acercamiento más pertinente puesto que permite abarcar la enfermedad en toda su complejidad (Donini et al., 2009). Los tratamientos actuales no se centran sólo en la educación sobre nutrición o el aumento de la actividad física, sino también en la consideración y evaluación de aspectos psicológicos asociados, ya que estos precipitan y perpetúan el consumo exagerado de alimentos, por lo que deben ser considerados si se pretende mantener un peso luego de haber alcanzado el ideal o deseable.

\section{EI Modelo Transteórico de motivación al cambio, enfoque terapéutico orientado al tratamiento de la obesidad}

El abordaje y tratamiento de las características psicológicas es de fundamental importancia, pues de lo contrario el paciente no es capaz de comprometerse adecuadamente con el tratamiento, asumiendo que en esta problemática hay factores de comportamiento que pueden debilitar el éxito del tratamiento (Cabello \& Zúñiga, 2007). Las personas con sobrepeso u obesidad se benefician con las intervenciones psicológicas y particularmente con estrategias cognitivas conductuales para mejorar la reducción de peso. Son predominantemente útiles cuando se combinan con estrategias dietéticas y de ejercicio.

De acuerdo a Shaw, O'Rourke, Del Mar y Kenardy (2005), son varios los métodos psicológicos que se usan para procesar y ayudar a perder peso en las personas que padecen sobrepeso u obesidad, los que pueden ser realizados de forma individual y/o grupal. Estos tratamientos implican un cambio global del estilo de vida, puesto que los logros a largo plazo requieren la modificación de actitudes, creencias y comportamientos acerca de la alimentación y la actividad física (Hill
\& Wyatt, 2002); todo ello supone un proceso activo, en el que la persona deberá hacer un esfuerzo considerable para cambiar hábitos perpetuados en el tiempo (Rollnick, 1996), motivo por el cual los tratamientos en obesidad no siempre obtienen buenos resultados.

Para Cabrera (2000), un problema asociado a lo anterior es que el comportamiento con efectos en salud "ha sido observado, descrito y explicado como un evento discreto, más que como un proceso y mucho menos como una secuencia de etapas" (p. 3). Esto implica que no se consideran las características específicas del grupo a las que se han dirigido las intervenciones.

Uno de los enfoques terapéuticos -a nivel psicológico-ampliamente utilizado es el Modelo Transteórico de motivación al cambio (Prochaska \& Velicer, 1997), descrito como un marco de intervenciones efectivas para promover cambios de conductas en salud a partir de las características específicas de los grupos a quienes están dirigidas las acciones (Cabrera, 2000). El modelo obtuvo su nombre de la integración de los principios y componentes teóricos de los diferentes sistemas de intervención analizados.

El modelo está fundado en la premisa básica de que el cambio comportamental es un proceso y que las personas tienen diversos niveles de motivación, de intención de cambio. Pero, entre otras premisas, Prochaska y Velicer (1997) señalan que el cambio es un proceso que se presenta como una secuencia de etapas estables, pero abiertas al cambio. Además, plantean que intervenciones no orientadas según estas etapas, los grupos permanecerán estancados, sin motivación, ni intención de participar en las intervenciones o programas ofrecidos. Finalmente, el reto del modelo, es avanzar hacia programas e intervenciones de la salud comportamental, de base poblacional, con apoyo de estrategias interactivas que incluyan un soporte ambiental a los cambios (Cabrera, 2000).

Desde este enfoque se sostiene que, en la mayoría de las personas, los cambios ocurren en forma gradual a través del tiempo y se pasa progresivamente por las siguientes "Etapas de Cambio": a) Pre-contemplación: la persona no está motivada; no hay intenciones de hacer modificaciones en los próximos 6 meses; b) Contemplación: hay pensamiento sobre posibles cambios en el comportamiento en los próximos 6 meses; c) Preparación para la acción: planificación activa para cambiar el comportamiento en un plazo definido, por ejemplo en 30 días; d) Acción: desarrollo de nuevos comportamientos; e) Mantenimiento: mantención del comportamiento modificado por seis meses o más; f) Terminación: llegar a un cambio sostenible y de 
confianza en sí mismo para no volver a las etapas anteriores.

En este marco, el modelo de motivación en espiral propone que los individuos pueden avanzar y retroceder en estas etapas de cambio antes de alcanzarlo. Como eje del proceso, la motivación cumple un papel fundamental; es el impulso que inicia, guía y mantiene el comportamiento, hasta alcanzar la meta u objetivo deseado (Prochaska \& DiClemente, 2002). El trabajo sobre motivación, ansiedad y depresión constituyen elementos fundamentales en la interacción y el acercamiento subjetivo a la realidad en los distintos ciclos vitales, variables a utilizar en el tratamiento psicológico del programa (Prochaska, Wright \& Velicer, 2008).

Este modelo ha sido amplia y efectivamente utilizado en el ámbito general de la salud; por ejemplo, en el tratamiento para disminución de la adicción al tabaco (Schumann et al., 2007), la promoción de actividad física en pacientes con diabetes y enfermedades cardiovasculares (Kirk et al., 2010), sintomatología depresiva en adolescentes (Lewis et al., 2009), alcoholismo (Demmel, Beck, Richter \& Reker, 2004), abuso de drogas (Henderson, Galen \& Saules, 2004). En el tratamiento de la obesidad igualmente se han presentado resultados favorables (Dray \& Wade, 2012; Johnson et al., 2008; Kirk et al., 2010).

En Chile, el Modelo Transteórico de motivación al cambio se utiliza terapéuticamente con frecuencia (Besser \& Moncada, 2013), modificando, de acuerdo a las características de los destinatarios, los énfasis teóricos y la regulación temporal del tratamiento. En este artículo se presentan-desde el punto de vista de la psicología- los efectos sobre la sintomatología ansiosa y depresiva de pacientes de la Agrupación de Obesidad Mórbida (OBEMOB) que participaron de un programa interdisciplinar.

El modelo transteórico ha sido complementado con los aportes de la psicoterapia constructivista, en particular el enfoque posracionalista cognitivo procesal sistémico de Vittorio Guidano (1983; 1999), enfatizando los procesos de regulación emocional y autoobservación. Este es un modelo de intervención centrado en el proceso de construcción de la identidad, donde la persona es el agente regulador central de la organización autoreferencial del conocimiento y su experiencia, otorgándole a la emotividad y subjetividad una importancia relevante en la psicoterapia. Los vínculos y conexiones emocionales sedimentan el funcionamiento psicológico de las personas, articulando los procesos tácitos y explícitos del conocimiento en la construcción de significado, definiendo de esta forma un sentido de sí mismo y del mundo (Arciero, 2005; Guidano, 1987, 1991, 1995).

\section{Metodología}

\section{Participantes}

Participaron 18 personas, todos miembros del grupo de OBEMOB, dependiente de la Municipalidad de Temuco que, a la vez, fueron parte del programa de apoyo multidisciplinario para la obesidad mórbida de la Universidad Santo Tomás, sede Temuco. Los participantes no han formado parte de programas de tratamientos para la obesidad y todos proceden de la zona urbana. La participación en el programa fue voluntaria, siendo el único criterio de ingreso el presentar la enfermedad, sin realizar un proceso previo de selección. Las edades de los participantes fluctuaron entre los 20 y 60 años, $(M=39.5 ; \mathrm{DT}=9.22)$, siendo 17 mujeres y 1 hombre. El programa comenzó una vez que todos los participantes estuvieron inscritos, de modo que todos quienes fueron inicialmente evaluados en ansiedad y sintomatología depresiva, se mantuvieron hasta finalizar el programa.

\section{Instrumentos}

Inventario de Ansiedad Rasgo/Estado (IDARE) [The State-Trait Anxiety Inventory (STAI)] de Spielberger, Gorsuch y Lushene (1970). Es un inventario diseñado para evaluar dos formas relativamente independientes de la ansiedad que constituyen dos subescalas con 20 ítems cada una: la ansiedad como estado (condición emocional transitoria), que incluye ítems como "Me siento seguro" y la ansiedad como rasgo (propensión ansiosa relativamente estable), que incluye ítems como "Veo que las dificultades se amontonan y no puedo con ellas". Cada una de ellas tiene 20 ítems; la escala estado incluye 10 ítems positivos de ansiedad (o sea, que a mayor puntuación mayor ansiedad) y 10 ítems negativos. La escala rasgo tiene 13 ítems positivos y 7 negativos.

El IDARE es un cuestionario autoaplicado, la forma de respuesta es de tipo Likert, que oscila entre 0 a 4 en ambas subescalas, en la escala de estado: (1-no totalmente, 2-un poco, 3-bastante, 4-mucho); en la escala rasgo (1-casi nunca, 2-algunas veces, 3 -frecuentemente, 4-casi siempre). La puntuación se obtiene de la suma de los ítems de cada dimensión, considerando 
las puntuaciones inversas, lo que entrega puntajes de ansiedad para cada escala.

El trabajo actual con obesos mórbidos, a nivel psicológico, nos entrega la experiencia de poder utilizar de manera psicoterapéutica y cualitativa los síntomas, como una forma de examinar y reflexionar sobre los cambios en la sintomatología, percibiendo más de lo que experimenta y experimentar más que aquello a lo que atiende conscientemente (Guidano, 1987). Los síntomas positivos y negativos que la aplicación del instrumento entrega, son trabajados con los pacientes durante el programa, a través de una constante reevaluación, y re-significación emocional de los eventos (Gross, 2002); de modo que el STAI no solo permite identificar la sintomatología, sino también operar como un criterio de contraste.

El instrumento ha mostrado adecuados indicadores de validez interna en distintos contextos de aplicación, por ejemplo, Guillén y Buela (2011) señalaron puntajes alfa de .90 para ansiedad rasgo y .94 para la dimensión ansiedad estado; en Chile el instrumento fue validado para adolescentes y adultos por Vera, Celis, Córdova, Buela y Spielberger (2007), quienes presentan buenos indicadores de confiabilidad (.92 para la muestra total y .87 para los rasgos) y validez. De la misma forma, la escala ha sido ampliamente utilizada en contextos de salud, habitualmente para probar eficacia de tratamientos (González, Sánchez, Sánchez \& Morales, 2007) y en descripciones de pacientes obesos (Mancilla, 1992). En la presente investigación se obtuvieron indicadores alfa de 0,84 y 0,80 para las dimensiones positiva y negativa de la escala de Ansiedad situacional e indicadores alfa de 0,79 y 0,72 para las dimensiones positiva y negativa de la escala de Ansiedad rasgo.

Dass 21: El DASS - 21, es un instrumento originalmente diseñado para evaluar la sintomatología depresiva, ansiedad y estrés en estudiantes universitarios. Ha sido validado en Chile por Antúnez y Vinet (2012). El instrumento se encuentra organizado en tres factores que corresponden a las dimensiones señaladas previamente (depresión, ansiedad y estrés). Es un instrumento auto-aplicado de 21 ítems, con cuatro alternativas de respuesta en formato Likert, que varían desde 0 ("No describe nada de lo que me pasó o sentí en la semana") hasta 3 ("Sí, esto me pasó mucho, o casi siempre"). El instrumento ha sido utilizado frecuentemente para evaluar sintomatología depresiva en pacientes OBEMOB (Aguilar, Manrique, Tuesta \& Musayón, 2010), presentando adecuadas propiedades psicométricas, por ejemplo, el estudio de Apostolo et al. (2006), señaló índices alfa de Cronbach de 0.90 para la depresión, 0.86 para la ansiedad, 0.88 para el stress y un ajuste aceptable a un modelo de tres factores en muestras de habla hispana (Bados et al., 2005; Daza et al., 2002; Román, 2010). Los índices de confiabilidad interna en la presente investigación, correspondieron a .81 para la escala de depresión; .83 para la escala de ansiedad y .85 para la escala de estrés.

\section{Diseño y procedimiento}

Se implementó un diseño cuasi-experimental con medidas pre-post, no contando con un grupo de cuasi-control (Cook \& Campbell, 1979). El contacto con la agrupación de obesos mórbidos perteneciente a la municipalidad de Temuco, se realizó a través del programa OBEMOB de la Universidad Santo Tomás, sede Temuco. Los participantes del grupo cuasiexperimental accedieron a integrarse a un programa multidisciplinar que incluyó un trabajo integrado con nutricionista, kinesiólogo, profesor de educación física y psicólogo. Cada área trabajó aproximadamente 8 horas semanales. En el área de la psicología, se trabajó durante 10 meses, con sesiones grupales de manera semanal, así como con sesiones individuales semanales. La aplicación de los instrumentos fue de manera grupal, 5 días antes de comenzar el tratamiento y 5 días posteriores al término del programa, la aplicación del instrumento duró aproximadamente 25 minutos.

La intervención psicológica estuvo orientada desde el Modelo Transteórico de motivación al cambio (Prochaska \& Velicer, 1997) previamente descrito. Desde una perspectiva de la psicología clínica se realizó un programa de intervención para el logro de objetivos terapéuticos orientado a la disminución de los síntomas ansiosos y depresivos presentes en pacientes con obesidad mórbida.

En un primer paso se identifica el nivel de la sintomatología presente en todos los participantes del programa para luego realizar entrevista clínicas de motivación al cambio, visualizando de esta forma la "fase del cambio" en que se encuentra cada uno de los participantes, en relación al compromiso con el tratamiento y los cambios de conductas que deben realizar para mejorar su calidad de vida.

El tratamiento consta de sesiones de grupo e individuales. El promedio de sesiones grupales es de 20 y 5 sesiones individuales por participante. Se entrega a los participantes una comprensión cognitiva post racionalista de los trastornos alimentarios, específicamente de la obesidad, así como de la sintomatología ansiosa y depresiva.

Se utilizaron técnicas cognitivas de revisión mental (reevaluación), reestructuración cognitiva, 
entrenamiento en solución de problemas, técnicas de respiración y relajación progresiva. Como hemos señalado previamente, se complementó el Modelo Transteórico con el enfoque cognitivo-posracionalista, enfatizando los procesos de autoobservación en la regulación personal. En todo el proceso terapéutico se evalúa el grado motivacional, permitiendo visualizar las dificultades y proyecciones para la adherencia al tratamiento. La emotividad y la subjetividad como hilo conductor en el proceso psicoterapéutico, orientando la mirada del paciente hacia la comprensión de su propio estilo afectivo y de las reglas de coherencia interna que constituyen el origen de la sintomatología clínica, este proceso constante de autoobservación (metodología), pretende lograr que el paciente amplíe el abanico de posibilidades y la conciencia que tiene de su funcionamiento emocional y cognitivo, articulando más los modelos de sí mismo y de la realidad que posee (Guidano, 1987; 1994).

\section{Resultados}

El análisis estadístico fue llevado a cabo utilizando estadísticos de comparación de grupo. Teniendo en consideración el bajo número de participantes, así como a las características de la distribución de las variables dependientes, donde ninguna se ajusta a una distribución normal $(\mathrm{S}-\mathrm{W}<0.05)$, es que se utilizaron pruebas no paramétricas de comparación, en particular, la prueba de los rangos con signo de Wilcoxon (W de Wilcoxon), junto a estadísticos descriptivos.

El el análisis previo, se contrastaron las variables edad y género con cada una de las variables dependientes, no observándose relaciones (en el caso de la edad) o efectos estadísticamente significativos en el caso del género.

Los resultados para la comparación de grupos (pre y post-test) se presentarán de manera separada para cada variable dependiente: ansiedad de estado, rasgo y depresión. La Tabla 1 muestra las medianas y desviaciones típicas para cada dimensión de la variable ansiedad en las mediciones pre y post-test.

Tabla 1

Estadísticos descriptivos ansiedad estado y rasgo

\begin{tabular}{lcccc}
\hline & \multicolumn{2}{c}{ Pre-Test } & \multicolumn{2}{c}{ Post-Test } \\
\cline { 2 - 5 } & Med. & Dt & Med. & Dt \\
\hline Ansiedad Estado & 45,1 & 9,9 & 37,2 & 9,1 \\
Ansiedad Rasgo & 40,2 & 6,8 & 37,1 & 8,9 \\
\hline
\end{tabular}

\section{Resultados para ansiedad de estado}

Los puntajes de la variable ansiedad de estado fueron calculados de acuerdo a las instrucciones de Spielberger, Gorsuch y Lushene (1982), desde donde se organizaron tres grupos según nivel de ansiedad: ansiedad de estado alto, medio y bajo. La comparación de proporciones evidencia variaciones con diferencias estadísticamente significativas, $\mathrm{Z}=-2,309 ; \mathrm{p}=.021$. Esto indica que, existen variaciones al comparar la distribución de las proporciones según el nivel de ansiedad de estado entre los grupos (pre y post tratamiento).

En la Tabla 1 se pueden observar la distribución de porcentajes, donde el nivel alto de ansiedad de estado pasa de incluir al 50\%, de los participantes, a incluir el $22,2 \%$ (5 personas menos) y el nivel bajo de ansiedad, pasa de incluir al 5,6\% de los participantes, a incluir al $22,2 \%$ ( 3 personas más).

Junto con comparar los cambios en las proporciones en el nivel de ansiedad de estado, se compararon los puntajes de esta escala en una medida pre y post (Med. Pre $=45.1$; Med.Post $=37,2$ ). La prueba de los rangos con signo de Wilcoxon entrega un valor $Z=-2,811$; $\mathrm{p}=0.005$, evidenciando una disminución estadísticamente significativa de la sintomatología evaluada. Ver Figura 1.

\section{Resultados para ansiedad de rasgo}

$\mathrm{Al}$ igual que en la ansiedad de estado, los puntajes de la variable ansiedad de rasgo fueron calculados de acuerdo a las instrucciones de Spielberger, Gorsuch y Lushene (1982), desde donde se organizaron tres grupos según nivel de ansiedad: ansiedad de rasgo alto, medio y bajo. La comparación de proporciones no evidencia variaciones con diferencias estadísticamente significativas, $\mathrm{Z}=-1,633 ; \mathrm{p}=.102$. Esto indica que, aunque existieron variaciones al comparar la distribución de las proporciones según el nivel de ansiedad de estado entre los grupos (pre y post tratamiento), estas no alcanzan a ser estadísticamente significativas.

En la Tabla 1 se pueden observar la distribución de porcentajes, donde el nivel alto de ansiedad de rasgo pasa de incluir al 22,2\%, de los participantes, a incluir el $16,7 \%$ (1 persona menos) y el nivel bajo de ansiedad, pasa de incluir al $11,1 \%$ de los participantes, a incluir al 27,8\% (3 personas más).

Junto con comparar los cambios en las proporciones en el nivel de ansiedad de rasgo, se compararon los puntajes de esta escala en una medida pre y post (Med. Pre $=40,2 ;$ Med.Post = 37,1). La prueba de los rangos 
con signo de Wilcoxon entrega un valor $Z=-1,851 ; p$ $=0.064$, de modo que, a pesar de la disminución en el rango promedio de la sintomatología evaluada, esta disminución no alcanza a ser estadísticamente significativa de la sintomatología evaluada. Ver Figura 1.

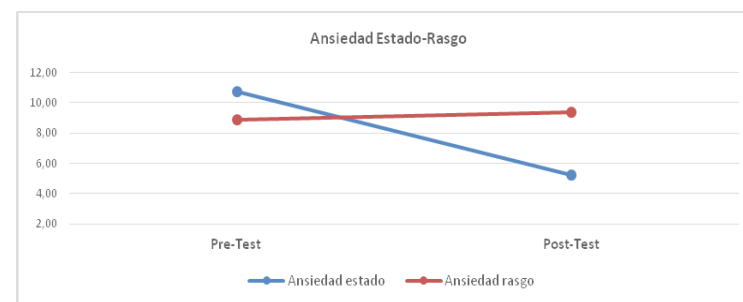

Figura 1:

Rangos promedios de las variables ansiedad estado y rasgo en sus medidas pre y post-test

\section{Resultados para las variables depresión, ansiedad y estrés incluidas en el instrumento DAS.}

La prueba de Wilcoxon conducida para estimar el impacto del programa en la dimensión sintomatología depresiva, indicó un efecto significativo del programa (Mpretest = 5.6; Mposttest = 2,8), Z = -3,107; $\mathrm{p}=.002$. La misma prueba, conducida para estimar el impacto del programa en la dimensión ansiedad, evidenciando una disminución estadísticamente significativa de la sintomatología evaluada $($ Mpretest $=5.7$, Mposttest $=$ 3.3), $Z=-2,255 ; p=.024$. En la dimensión estrés se observa una disminución estadísticamente significativa de la sintomatología evaluada $($ Mpretest $=7.3$, Mposttest $=$ 4.5), $Z=-3,280 ; p=.001$. Ver Figura 2 y Tabla 2.

Tabla 2

Estadísticos descriptivos DAS

\begin{tabular}{lcccc}
\hline & \multicolumn{2}{c}{ Pre-Test } & \multicolumn{2}{c}{ Post-Test } \\
& Med. & Dt & Med. & Dt \\
\hline Depresión & 5,6 & 3,9 & 2,8 & 2,2 \\
Ansiedad & 5,7 & 4,9 & 3,3 & 3,3 \\
Estrés & 7,3 & 4,4 & 4,5 & 3,2 \\
\hline
\end{tabular}

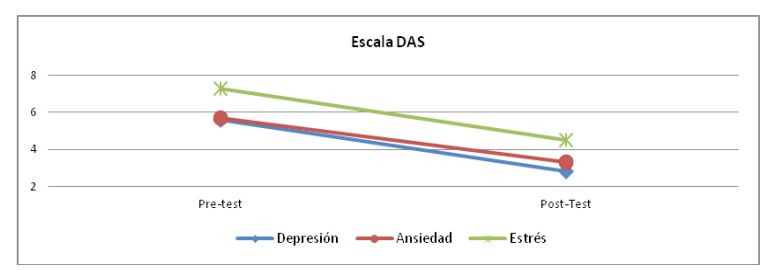

Figura 2:

Medias de las variables DAS en sus medidas pre y post-test

\section{Discusión y conclusiones}

Esta investigación pretendió evaluar los efectos de un programa interdisciplinario para el tratamiento de la obesidad, en particular sobre las dimensiones psicológicas asociadas a esta enfermedad. De esta forma, se orientó la intervención desde el marco del modelo transteórico de motivación al cambio (Prochaska \& DiClemente, 1982), así como desde las orientaciones específicas del modelo cognitivo posracionalista de Guidano (1991), evaluando la sintomatología ansiosa y depresiva. En el marco de una disminución de peso (Feixas \& SaldivarMaldonado, 2010), los resultados señalan un efecto positivo del tratamiento desde el ámbito psicológico que se tradujo en la reducción de sintomatología en todas las dimensiones evaluadas. Estos resultados concuerdan con una revisión teórica de los tratamientos psicológicos en obesos de Larragaña y Garcia-Mayor (2007), en la que señalan que es posible un cambio psicológico en estos pacientes, específicamente en el estilo de vida y la forma de enfrentarse a la enfermedad, a su vez validando los procesos psicoterapéuticos orientados al trabajo de las cogniciones y su individualidad.

Por su parte, Feixas y Saldivar-Maldonado (2010) evalúan la eficacia de la intervención psicológica en la reducción de la ansiedad en pacientes quirúrgicos de banda gástrica ajustable, utilizando dos instrumentos: a) El State-Trait Anxiety Inventory (STAI) en que sólo aplican la escala A-E, evaluando un estado emocional transitorio, que entrega información variable y modificable en el tiempo, ya que señalan que sería la sintomatología posible de disminuir; b) La escala EVA (Escala Visual Analógica), que mide el dolor subjetivo, la que además entrega información respecto a la presencia de sintomatología ansiosa. Por lo anterior es posible comparar nuestro estudio con el de Feixas y Saldivar-Maldonado (2010) desde la escala ansiedad estado, que mide sintomatología modificable. Es importante mencionar que la inclusión en el presente estudio de la escala ansiedad rasgo posibilita analizar características estables como perfil psicológico de los pacientes atendidos, variable que estaría implicada en el exceso de ingesta alimenticia.

Con relación a los resultados encontrados en este estudio sobre sintomatología ansiosa y depresiva en pacientes obesos, estos concuerdan con los descritos por Ríos et al., (2008), planteando la existencia de niveles de ansiedad y de depresión en obesos. Dichos niveles de sintomatología son mayores en mujeres, lo anterior necesariamente debe tenerse en cuenta en el tratamiento multidisciplinar de la obesidad. 
El estudio presenta algunas limitaciones metodológicas que es importante señalar, por ejemplo, el tamaño de la muestra no permite generalizar y comparar la investigación con otros estudios de forma favorable, por lo que en futuras investigaciones debiesen considerarse un número mayor de participantes. De la misma forma, futuras investigaciones debiesen considerar la posibilidad de utilizar un diseño de cuasi-control para tener un grupo de comparación que pueda mejorar la validez del estudio. Finalmente, es importante complementar los próximos estudios con medidas repetidas e integrar mediciones que también pueden estar relacionadas con la obesidad mórbida, como la regulación emocional y los estilos atributivos.

A pesar de las limitaciones mencionadas se considera que estos resultados pueden ser explicados desde al menos cuatro dimensiones: identificación de sintomatología, diferenciación emocional, atribuciones internas y externas, motivación asociada al apoyo social y sensación de pertenencia.

a) La identificación de sintomatología como parte de una forma regular de explicarse el mundo y la enfermedad: la comprensión de la obesidad, como parte de una forma regular de explicarse el mundo y de regulación emocional, formó parte de las 4 primeras sesiones, siendo psico-educativas sobre la enfermedad y sus consecuencias psicológicas en las que la ansiedad y depresión forman parte central. La comprensión de la obesidad como una enfermedad es entonces un elemento clave en la motivación al cambio y un primer paso en el proceso de autoobservación que implica tanto el reconocimiento de la sintomatología como de los procesos que la mantienen. La autoobservación permite la conexión con los propios procesos automantenidos que se hacen ahora conscientes, permitiendo la reevaluación del por qué comer y las consecuencias negativas del proceso (Silva, 2003).

b) Diferenciación emocional: en el marco de los procesos de autoobservación, no sólo son fundamentales aquellos asociados a la reevaluación cognitiva de las motivaciones vinculadas a la ingesta de alimentos, sino que también es clave una exploración profunda de las emociones vinculadas a la ingesta alimenticia. Este proceso implica autoobservación y reconocimiento de emociones básicas y cognitivas subyacentes que contribuyen no solo a la mantención de la enfermedad, sino también a una forma de sentirse en el mundo. En este punto, el trabajo psicológico ha contribuido a esta identificación emocional y, asociado a lo anterior, a una forma más flexible de reconocer y regular emociones. c) Atribuciones internas y externas: se considera que el cambio que ha permitido el reconocimiento de emociones, permite además identificar polos emocionales, descritos teóricamente como pasivos y activos (Guidano, 1987). En términos generales, a su ingreso al programa, los participantes mantienen una explicación pasiva de su rol en el mundo, las cosas les suceden y pueden hacer poco por cambiar este destino. El proceso de autoobservación llevado a cabo durante las sesiones, ha implicado el integrar un rol activo en el mundo, incorporándose como agente de cambio. Como hemos venido señalando, este proceso de flexibilización cognitiva requiere de una autoobservación constante, de identificación y reconocimiento de emociones discrepantes, así como dela integración de emociones que han mantenido una forma de ser y estar en mundo, en otras palabras, permite un aumento de los niveles de abstracción. El tener la oportunidad de considerarse como agentes activos (en oposición a un rol pasivo automantenido), permite re-construir un sentido de sí mismo, menos difuso y una identidad más estable y flexible (Guidano, 1995).

d) Motivación asociada al apoyo social y sensación de pertenencia: los procesos que hemos descrito previamente, tienen un claro énfasis en un trabajo individual a través de la autoobservación, asumiendo el terapeuta un rol de perturbador estratégicamente orientado. Sin embargo, los procesos de integración y pertenencia social son también fundamentales, principalmente porque mantienen la motivación al cambio y adherencia al tratamiento. Los procesos de autoobservación descritos previamente, son altamente complejos, consideramos que la presencia de afectos y emociones grupales aporta a una valoración positiva del trabajo individual (Gracia $\&$ Herrero, 2006), cada uno de los participantes, puede entonces asumir un rol de perturbador que complementa el rol del terapeuta, pero a la vez lo enriquece al ser un par que media y potencia el mejor uso de los recursos personales y sociales (Barra, 2004).

En resumen, consideramos que el programa entrega un contexto de pertenencia y apoyo social que permite una exploración segura de los procesos cognitivos y emocionales que mantienen una forma estable de ser en el mundo, favoreciendo además un aumento en los niveles de abstracción, lo que potencia un proceso básico de autoobservación. El apoyo social percibido permite alcanzar niveles de bienestar y a la superación de acontecimientos estresantes por medio de la valoración que una persona hace sobre su red social y los recursos que encuentra en ella. 
Como se ha señalado previamente, futuras investigaciones deben considerar muestras mayores de pacientes y realizar seguimientos para conocer la estabilidad de los resultados, asumiendo la necesidad de generar evidencia científica sobre el aporte que hacen este tipo de intervenciones, a su vez deben considerar la evaluación de indicadores clínicos que permitan incorporar información que oriente sobre los aspectos de la intervención que valoran los pacientes, así como el reporte de resultados a mediano y corto plazo. Finalmente, se piensa que este reporte aporta a una visión más integral del proceso de cambio tanto en las conductas que mantienen la sobrealimentación así como en la percepción del sí mismo.

\section{Referencias}

Aguilar, C. M., Manrique, R. L., Tuesta, M. M. \& Musayón, O. Y. (2010). Depresión y autoestima en adolescentes con obesidad y sobrepeso: un problema que pesa. Revista enfermería Herediana, 3(1), 49-54. Recuperado de http://www.upch.edu.pe/faenf/images/pdf/Revistas/2010/ enero/Depresion\%203_7.pdf

Álvarez, R. (1998). Obesidad y autoestima. México: McGraw-Hill Interamericana.

Antony, M., Bieling, P., Cox, B., Enns, M. \& Swinson, R. (1998). Psychometric properties of the 42-item and 21-item versions of the Depression Anxiety Stress Scales in clinical groups and a community sample. Psychological Assessment, 10(2), 176-181. doi:10.1016/j. jad.2008.01.023

Antúnez, Z. \& Vinet, E. (2012). Escalas de Depresión, Ansiedad y Estrés (DASS - 21): Validación de la Versión abreviada en Estudiantes Universitarios Chilenos. Terapia psicológica, 30(3), 49-55. Recuperado de http://dx.doi.org/10.4067/S0718-48082012000300005

Apóstolo, J., Mendes, A. \& Azeredo, Z. (2006). Adaptación para la lengua portuguesa de la depression, anxiety and stress scale (DASS). Revista Latino-Americana de Enfermagem, 14(6), 863-871. Recuperado de http://dx.doi.org/10.1590/S0104-11692006000600006

Arciero, G. (2005). Estudio y Diálogos Sobre la Identidad Personal. Buenos Aires: Editorial Amorrortu.

Bados, A., Solanas, A. \& Andrés, R. (2005). Psychometric properties of the Spanish version of Depression, Anxiety and Stress Scales (DASS). Psicothema, 17(4), 679-683. Recuperado de http://www.psicothema. com/pdf $/ 3165$.pdf

Barra, E. (2004). Apoyo social, estrés y salud. Psicología y Salud, 14(2), 237-243. Recuperado de revistas.uv.mx/index.php/psicysalud/article/ download/848/1562

Besser, M. \& Moncada, L. (2013). Proceso psicoterapéutico desde la perspectiva de Terapeutas que tratan trastornos alimentarios: un estudio cualitativo. Psykhe, 22(1), 69-82. Recuperado de http://dx.doi. org/10.7764/psykhe.22.1.633

Black, D., Goldstein, R. \& Mason, E. (2003). Psychiatric diagnosis and weight loss following gastric surgery for obesity. Obesity Surgery, 13(5), 746-751.

Braguinsky, J., Markman, C. \& Katz, S. (1995). Una señal de Alarma: El aumento de Prevalencia de la obesidad. Nutrición Clínica, 2, 29-32.

Bresh, S. (2006). La obesidad: aspectos psicológicos y conductuales. Revista Colombiana de Psiquiatría, 35(4), 537-546. Recuperado de http://www.redalyc.org/pdf/806/80635407.pdf

Cabello, G. M. \& Zúñiga, Z. J. (2007). Aspectos intrapersonales y familiares asociados a la obesidad: un análisis fenomenológico. Ciencia UANL, 1O(2), 183-188. Recuperado de http://eprints.uanl.mx/1770/1/ OBESIDAD.pdf
Cabrera, G. (2000). El modelo transteórico del comportamiento en salud. Revista Facultad Nacional de Salud Pública, 18(2), 129-138. Recuperado de http://www.redalyc.org/pdf/120/12018210.pdf

Castellanos, B., Grau, J. \& Martín, M. (1986). Caracterización de la ansiedad personal presente en la personalidad premórbida de pacientes con trastornos transitorio situacionales y neurosis de ansiedad. Reporte de investigación, Facultad de Psicología de la Universidad de la Habana, Cuba.

Cook, T. D. \& Campbell, D. T. (1979). Quasi-Experimentation Design and Analysis Issues for Fields Settings. Chicago: Rand McNally.

Daza, P., Novy, D., Stanley, M. \& Averill, P. (2002). The depression anxiety stress scale-21: Spanish translation and validation with a hispanic sample. Journal of Psychopathology and Behavioral Assessment, 24(3), 195-205. Recuperado de http://dx.doi.org/10.1023/A:1016014818163

De Chouly De Lenclave, M. B., Florequin, C. \& Bailly, D. (2001). Obesity, alexithymia, psychopathological disorders, and binge eating: a comparative study between 40 obese subjects and 32 controls. L'Encephale, 27, 343-350. Recuperado de http://www.researchgate. net/publication/11667221_Obesity_alexithymia_psychopathological_ disorders_and_binge_eating_A_comparative_study_between_40_obese_subjects_and_32_controls

Demmel, R., Beck, B., Richter, D. \& Reker, T. (2004). Readiness to change in a clinical sample of problem drinkers: Relation to alcohol use, self-efficacy and treatment outcome. European Addiction Research, 10(3), 133-138. doi:10.1159/000077702

Donini, L. M., Savina, C., Castellaneta, E., Coletti, C., Paolini, M., Scavone, L., ... \& Cannella, C. (2009). Multidisciplinary approach to obesity. Eat \& Weight Disorders, 14(1), 23-32. Recuperado de http:// link.springer.com/article/10.1007\%2FBF03327791\#/page-1

Dray, J. \& Wade, T. D. (2012). Is the transtheoretical model and motivational interviewing approach applicable to the treatment of eating disorders? A review. Clinical Psychology Review, 32(6), 558-565. doi:10.1016/j. cpr.2012.06.005

Elfhag, K., Carlsson, A. M. \& Rossner, S. (2003). Subgrouping in obesity based on Rorschach personality characteristics. Scand J Psychol, 44(5), 399-407.

Feixas, G. \& Saldivar-Maldonado, P. (2010). Eficacia de la intervención psicológica en la reducción de la ansiedad en pacientes quirúrgicos de banda gástrica. Revista Boletín de Psicología, 99, 71-87. Recuperado de http://www.uv.es/seoane/boletin/previos/N99-4.pdf

Franco, A., Martín, M. \& Grau, J. (1992). Las contradicciones internas de la personalidad en la génesis de los estados de tensión emocional. Reporte de investigación, Facultad de Psicología de la Universidad de la Habana, Cuba.

Frelut, M.L. (2009). Obesidad del niño y el adolescente. EMC-Pediatría, 44(4), 1-15. doi:10.1016/S1245-1789(09)70187-8

Gracia, E. \& Herrero, J. (2006). La comunidad como fuente de apoyo social. Evaluación e implicaciones en los ámbitos individual y comunitario. Revista Latinoamericana de Psicología, 38(2), 327-342. Recuperado de http://www.scielo.org.co/pdf/rlps/v38n2/v38n2a07.pdf

Gross, J. J. (2002). Emotion regulation: Affective, cognitive, and social consequences. Psychophysiology, 39(3), 281-291. Recuperado de http://onlinelibrary.wiley.com/store/10.1017/S0048577201393198/ asset/S0048577201393198.pdf?v=1\&t=igbfg53k\&s=7e488b4e9b07 $718348 c 3 e 61450 \mathrm{dc} 9 \mathrm{dbbbafc0c10}$

Guidano, V. F. (1999). Psicoterapia: Aspectos metodológicos, cuestiones clínicas y problemas abiertos desde una perspectiva post-racionalista. Revista Psicoterapia, 10(37), 95-105.

Guidano, V. F. (1991). The self in process: Toward a post-rationalist cognitive therapy. New York: Guilford Press.

Guidano, V. F. (1995). Desarrollo de la terapia cognitiva posracionalista. Santiago de Chile: Instituto de Terapia Cognitiva.

Guidano, V. F. (1999). Psicoterapia: Aspectos metodológicos, cuestiones clínicas y problemas abiertos desde una perspectiva post-racionalista. Revista Psicoterapia. 37, 95-105.

Guidano, V.F. \& Liotti, G. (1983). Cognitive processes and emotional disorders. New York: Guilford.

Guillén-Riquelme, A. \& Buela-Casal, G. (2011). Actualización psicométrica y funcionamiento diferencial de los ítems en el State Trait 
Anxiety Inventory. Psicothema, 23(3), 510-515. Recuperado de http:// www.psicothema.com/pdf/3916.pdf

Halsam, D. \& James, P. (2005). Obesity. The Lancet, 366, 1197-1209. doi:10.1016/S0140-6736(05)67483-1

Henderson, M. J., Galen, L.W. \& Saules, K. (2004). The predictive validity of the University of Rhode Island change assessment questionnaire in a heroin-addicted polysubstance abuse sample. Psychology of Addictive Behaviours, 18(2), 106-112. Recuperado de http://dx.doi. org/10.1037/0893-164X.18.2.106

Hill, J. \& Wyatt, H. (2002).Outpatient management of obesity: a primary care perspective. Obesity. Research, 10(2), 124-130.

Hollis, J. (2005). La obesidad es un problema familiar. México: Promexa. Johnson, S., Paiva, A., Cummins, C., Johnson, J., Dyment, S., Wright, J., ... \& Sherman, K. (2008). Transtheoretical Model-based multiple behavior intervention for weight management: Effectiveness on a population basis. Preventive Medicine, 46(3), 238-246. doi:10.1016/j. ypmed.2007.09.010

Kannel, W., D'Agistino, R. \& Cobb, J. (1996). Effect of weight on cardiovascular disease. American Journal of Clinical Nutrition, 63(3), 419-422.

Kirk, A., MacMillan, F. \& Webster, N. (2010). Application of the Transtheoretical model to physical activity in older adults with Type 2 diabetes and/or cardiovascular disease. Psychology of Sport and Exercise, 11(4), 320-324. doi:10.1016/j.psychsport.2010.03.001

Komlos, J., Breitfelder, A. \& Sunder, M. (2009). The transition to postindustrial BMI values among US children. American Journal of Human Biology, 21(2), 151-160. doi:10.1002/ajhb.20806

Larrañaga, A. \& García-Mayor, R. (2007). Tratamiento psicológico de la obesidad. Medicina clínica, 129(10), 387-391. doi:10.1157/13110213

Lewis, C., Simons, A. D., Rohde, P., Silva, S. G., Small, D. M., Murakami, J. L., ... \& March, J. S. (2009). The role of readiness to change in response to treatment of adolescent depression. Journal of Consulting and Clinical Psychology, 77(3), 422-428. doi:10.1037/a0014154

Mancilla-Díaz, J. M., Duran-Díaz, A., Ocampo-Tellez-Giron, M.T., \& López-Alonso, V. (1992). Rasgos de personalidad comunes en obesos. Revista Salud Pública, 34(5), 533-539. Recuperado de http://redalyc. org.www.redalyc.org/articulo.oa?id=10634508

Martínez, B., Rodríguez, M. \& Martínez, J. (2003). Síndrome metabólico, resistencia a la insulina y metabolismo tisular. Endocrinología y Nutrición, 50(8), 324-333. doi:10.1016/S1575-0922(03)74546-X

Martínez-González, M. Á., Alfredo-Martinez, J., Hu, F. B., Gibney, M. J. \& Kearney, J. (1999). Physical inactivity, sedentary lifestyle and obesity in the European Union. International Journal of Obesity \& Related Metabolic Disorders, 23(11), 1192-1201. doi:10.1038/sj.ijo.0801049

Moreno, M. (2005). Obesidad, uno de los principales problemas de salud en el mundo. Ciencia, Conocimiento, Tecnología, 13, 34-35.

Nicklas, T., Baranowski, T., Cullen, K. \& Berenson, G. (2001).Eating patterns, dietary quality and obesity. J Am Coll Nutr, 20(6), 599-608. doi:10.1080/07315724.2001.10719064

O’Brien, P. E. \& Dixon, J. B. (2002). The extent of the problem of obesity. The American Journal of Surgery, 184(6), 4-8. doi:10.1016/ S0002-9610(02)01172-8

Peña, M. \& Bacallao, J. (2001). La obesidad y sus tendencias en la Región. Revista Panamericana de Salud Pública, 10(2), 45-78. doi:10.1590/ S1020-49892001000800001

Prochaska, J. \& DiClemente, C. (1982). Transactional therapy: toward a more integrative model of change. Psychotherapy: theory, research and practice, 19(3), 276-288. Recuperado de https://www.deepdyve. $\mathrm{com} / \mathrm{lp} / \mathrm{psycarticles}-\mathrm{reg} /$ transtheoretical-therapy-toward-a-moreintegrative-model-of-change-c76zXPn7tM

Prochaska, J. \& DiClemente, C. (2002). Transtheoretical Therapy. En F. W. Kaslow \& J. Lebow (Eds.), Comprehensive Handbook of Psychotherapy (vol. 4). New York: John Wiley \& Sons, Inc.

Prochaska, J. \& Velicer, W. (1997). The transtheoretical model of health behavior change. American Journal of Health Promotion, 12(1), 38-48. doi:10.4278/0890-1171-12.1.38

Prochaska, J. O., Wright, J. A. \& Velicer, W. F. (2008). Evaluating Theories of Health Behavior Change: A Hierarchy of Criteria Applied to the
Transtheoretical Model. Applied Psychology: An International Review, 57(4), 561-588. doi:10.1111/j.1464-0597.2008.00345.x

Puhl, R. \& Heuer, C. (2009). The stigma of obesity: A review and update. Obesity, a research journal, 17(5), 941-964. doi:10.1186/1471-245811-661

Pulido-Castelblanco, D., Novoa-Gómez, M. \& Muñoz-Martínez, A. (2013). Análisis metacontingencial de la obesidad mórbida como problemática de salud pública. Terapia psicológica, 31 (2), 239-247. Recuperado de http://www.redalyc.org/articulo.oa?id=78526609005

Pupo, O., Martin, M. \& Lopez, M. L. (1989). Ansiedad personal y calidad de la ejecución en sujetos normales y con disfunción sexual eréctil. Reporte de investigación, Facultad de Psicología de la Universidad Central, Chile.

Reyes, M., Díaz, E., Lera, L. \& Burrows, R. (2011). Ingesta y metabolismo energético en una muestra de adolescentes chilenos con sobrepeso y obesidad. Revista médica de Chile, 139(4), 425-431. doi:10.4067/ S0034-98872011000400002

Ríos, B., Rangel, G., Álvarez, R., Castillo, F., Ramírez, G. \& Pantoja, J. (2008). Ansiedad, depresión y calidad de vida en el paciente obeso. Acta Médica Grupo Ángeles, 6(4), 147-153. Recuperado de http:// www.medigraphic.com/pdfs/actmed/am-2008/am084a.pdf

Rollnick, S. (1996). Behaviour change in practice: targeting individuals. International Journal of Obesity, 20(1), 22-26.

Román, M. (2010). Validación de la versión abreviada de las escalas de depresión, ansiedad y estrés (DASS - 21) en adolescentes estudiantes de enseñanza media de la comuna de Temuco (Tesis de maestría inédita). Universidad de La Frontera, Temuco, Chile.

Rubenstein, A. H. (2005). Obesity: A modern epidemic. Transactions of the clinical an climatological association, 116, 103-113.

Sandoya, E., Schwedt, E., Moreira, V., Schettini, C., Bianchi, M. \& Senra, H. (2007).Obesidad en adultos: prevalencia y evolución. Revista Uruguaya de Cardiología. 22(2), 130-138. Recuperado de http://www. suc.org.uy/revista/v22n2/pdf/rcv22n2_8.pdf

Schumann, A., John, U., Ulbricht, S., Rüge, J., Bischof, G. \& Meyer, C. (2007). Variability of tailoring of a smoking cessation intervention based on the transtheoretical model. Addictive Behaviors, 32(12), 3083-3087. doi:10.1016/j.addbeh.2007.06.004

Shaw, K., O'rourke, P., Del Mar, C. \& Kenardy, J. (2005). Psychological interventions for overweigth. Cochrane Database of Systematic Reviews, 2(2). doi:10.1002/14651858.CD003818.pub2

Silva, J. (2003). Biología de la regulación emocional: Su impacto en la psicología del afecto y la psicoterapia. Terapia Psicológica, 21(2), 163-172. Recuperado de http://teps.cl/files/2011/06/ cap07.pdf

Silva, J. (2008). Sobrealimentación Inducida por la Ansiedad, Parte II: Un Marco de Referencia Neurocientífico para el Desarrollo de Técnicas Psicoterapeuticas y Programas de Prevención. Terapia Psicológica, 26(1), 99-115. Recuperado de http://dx.doi. org/10.4067/S0718-48082008000100009

Spielberger, C. D., Gorsuch, R. L. \& Lushene, R. E. (1982). Manual del Inventario de Ansiedad Estado-Rasgo. Madrid: TEA.

Vera-Villarroel, P., Celis-Atenas, K., Córdova-Rubio, N., BuelaCasal, G. \& Spielberger, Ch. (2007). Preliminary Analysis and Normative Data of the State-Trait Anxiety Inventory (STAI) in Adolescent and Adults of Santiago, Chile. Terapia Psicológica, 25(2), 155-162. Recuperado de http://teps.cl/files/2011/05/ scp7444-Terapia-Part61.pdf

Vernay, M., Malon, A., Oleko, A., Salanave, B., Roudier, C., Szego, E., ... \& Castetbon, K. (2009). Association of socioeconomic status with overall overweight and central obesity in men and women: the French Nutrition and Health Survey 2006. BMC Public Health, 9(1), 215-223. doi:10.1186/1471-2458-9-215

Wardle, J., Williamson, S., Johnson, F. \& Edwards, C. (2006). Depression in adolescent obesity: Cultural moderators of the depressive symptoms. International Journal of Obesity, 30(4), 634-643. doi: 10.1038/sj.ijo.0803142 